J. H. Khushvaktov ${ }^{1,2, *}$, J. Adam ${ }^{1,3}$, A. A. Baldin ${ }^{1,4}$, V. V. Chilap ${ }^{5}$, V. I. Furman ${ }^{1}$, F. Sagimbaeva ${ }^{1}$, A. A. Solnyshkin ${ }^{1}$, V. I. Stegailov ${ }^{1}$, P. Tichy ${ }^{1}$, V.M. Tsoupko-Sitnikov ${ }^{1}$, S. I. Tyutyunnikov ${ }^{1}$, R. Vespalec ${ }^{1}$, J. Vrzalova ${ }^{1,3}$, B. S. Yuldashev ${ }^{1,2}$, V. Wagner ${ }^{3}$, L. Zavorka ${ }^{1}$, M. Zeman ${ }^{1,6}$

\title{
INTERACTIONS OF SECONDARY PARTICLES WITH THORIUM SAMPLES IN THE SETUP QUINTA IRRADIATED WITH 6-GeV DEUTERONS
}

Submitted to "Nuclear Instruments and Methods in Physics Research B"

\footnotetext{
${ }^{1}$ Joint Institute for Nuclear Research, Dubna

${ }^{2}$ Institute of Nuclear Physics, AS RU, Tashkent

${ }^{3}$ Nuclear Physics Institute of the CAS, Czech Republic

${ }^{4}$ Institute for Advanced Studies "OMEGA", Dubna, Russia

${ }^{5}$ Center of Physical and Technical Projects "Atomenergomash", Moscow

${ }^{6}$ Brno University of Technology, Brno, Czech Republic

*E-mail: khushvaktov@jinr.ru
} 
Хушвактов Ж. Х. и др.

Взаимодействие вторичных частиц с образцами тория

на установке «Квинта» при облучении 6-ГэВ дейтронами

Сборка из природного урана (512 кг) «Квинта» облучалась 6-ГэВ дейтронами. Образцы ${ }^{232}$ Тh были расположены на центральной оси установки «Квинта». При анализе спектров гамма-лучей, испущенных облученными образцами ${ }^{232} \mathrm{Th}$, было идентифицировано более ста радионуклидов продуктов реакций, в том числе и ${ }^{233} \mathrm{U}$. Определено отношение массы образующегося ${ }^{233} \mathrm{U}$ к массе ${ }^{232} \mathrm{Th}$. Результаты эксперимента сравниваются с модельными расчетами по методу Монте-Карло (код FLUKA).

Работа выполнена в Лаборатории ядерных проблем им. В.П.Джелепова ОИЯИ.

Препринт Объединенного института ядерных исследований. Дубна, 2016

Khushvaktov J. H. et al.

E6-2016-62

Interactions of Secondary Particles with Thorium Samples

in the Setup QUINTA Irradiated with 6-GeV Deuterons

The natural uranium assembly, QUINTA, was irradiated with 6-GeV deuterons. The ${ }^{232}$ Th samples were placed at the central axis of the setup QUINTA. The spectra of gamma rays emitted by the activated ${ }^{232} \mathrm{Th}$ samples have been analysed, and more than one hundred nuclei produced have been identified. For each of those products, reaction rates have been determined. The ratio of the weight of produced ${ }^{233} \mathrm{U}$ to that of ${ }^{232} \mathrm{Th}$ is presented. Experimental results were compared with the results of Monte Carlo simulations by the FLUKA code.

The investigation has been performed at the Dzhelepov Laboratory of Nuclear Problems, JINR. 


\section{INTRODUCTION}

Accelerator Driven Systems (ADSs) are suggested as a means for safe, costeffective energy production and nuclear waste transmutation [1-4]. An ADS consists of a high-current, high-energy accelerator coupled with a subcritical nuclear assembly. During the past several years, such studies have been conducted with accelerated particle beams at the Nuclotron of the Joint Institute for Nuclear Research (JINR) in the framework of the international collaboration "Energy plus Transmutation of Radioactive Waste". This program has carried out a large number of experiments with the subcritical uranium target QUINTA [5-12], as well as the lead-graphite target GAMMA-3 $[13,14]$. Several experiments were conducted using a solid lead target GENERATOR [15-17] at the proton beam of the JINR Phasotron accelerator. ${ }^{232} \mathrm{Th}$ is a fissionable material by fast neutrons, but neutron capture reaction is also important in order to study the breeding efficiency of fissile ${ }^{233} \mathrm{U}$ in a thermal-spectrum ADS. In this paper we present new experimental data and a comparison with the calculations by the FLUKA $[18,19]$ code. For thorium samples, located at the central axis of the setup QUINTA, the reaction rates for generated radionuclides were measured and are presented in this paper.

\section{STRUCTURE OF THE SETUP QUINTA}

Uranium assembly QUINTA is presented in Fig. 1. It consists of five sections of hexagonal shaped aluminum containers with an inscribed circular hole of diameter $28.4 \mathrm{~cm}$. The containers are filled with cylindrical rods of natural uranium, having a sealed aluminum shell (external dimensions: $3.6 \mathrm{~cm}$ diameter, $10.4 \mathrm{~cm}$ in length, and $1.72 \mathrm{~kg}$ uranium mass). The containers are made of 5-mm-thick aluminum. The first section, facing the deuteron beam, contains 54 uranium rods and has a central beam window, $80 \mathrm{~mm}$ in diameter, installed in order to reduce its albedo and reduce the leakage of neutrons from the target. Four subsequent sections are structurally identical and contain 61 uranium rods. The mass of the natural uranium in each of these sections is $104.92 \mathrm{~kg}$, and the 


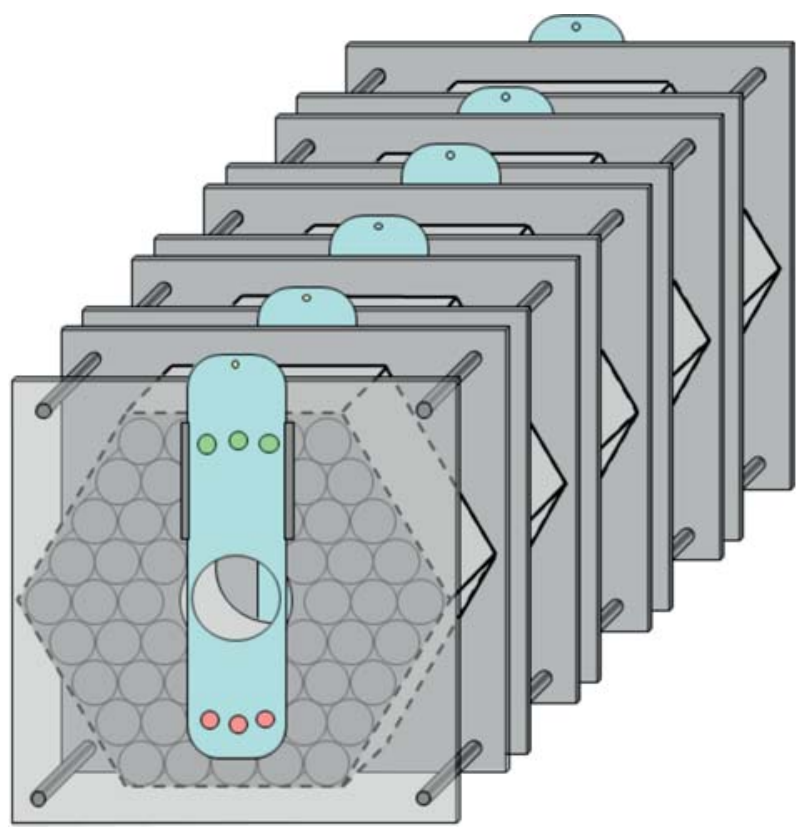

Fig. 1. General view of the QUINTA setup

total mass of uranium in the entire target is $512.56 \mathrm{~kg}$. The filling factor of the $2 \mathrm{nd}, 3 \mathrm{rd}, 4$ th, and 5 th uranium sections is about 0.8 , and is $\sim 0.6$ of the entire assembly.

\section{EXPERIMENT}

During the experiment, the ${ }^{232} \mathrm{Th}$ samples were conventionally marked as 9Th, 10Th, 11Th and 12Th, masses of the samples are respectively 0.1236 , $0.1242,0.1355$ and $0.1402 \mathrm{~g}$. The 9Th sample was placed on the central axis of the setup QUINTA, between the first and second sections at a distance of $12.1 \mathrm{~cm}$, the 10Th sample between the third and fourth sections at a distance of $25.2 \mathrm{~cm}$, the $11 \mathrm{Th}$ sample between the third and fourth sections at a distance of $38.3 \mathrm{~cm}$, and 12Th sample between the fourth and fifth sections at a distance of $51.4 \mathrm{~cm}$ from the front end. The total number of incident deuterons colliding with the target was determined by the standard method of activation of aluminum foil by the reaction ${ }^{27} \mathrm{Al}(d, x)^{24} \mathrm{Na}$. After 975 minutes irradiation the total number of deuterons incident on the target was $1.93(2) \mathrm{E}+13$. The technique used to determine the total number of incident deuterons on target is described in detail in Ref. [5]. The $\gamma$-ray spectra of the samples were measured with three 
HPGe detectors manufactured by ORTEC (two detectors with relative efficiencies of $28 \%$ and $33 \%$ and the energy resolutions of 1.9 and $1.8 \mathrm{keV}$, respectively, at the $1.33-\mathrm{MeV}^{60} \mathrm{Co}$ line) and CANBERRA (one detector with a relative efficiency of $19 \%$, and an energy resolution of $1.8 \mathrm{keV}$ at the $1.33-\mathrm{MeV}{ }^{60} \mathrm{Co}$ line). For each sample, from 8 to $13 \gamma$-ray spectra were measured with different time intervals and the cooling time of the first spectrum ranged from 120 to $160 \mathrm{~min}$. Energy and efficiency calibrations of the detectors were performed using a set of the $\gamma$-ray standards $\left({ }^{54} \mathrm{Mn},{ }^{57} \mathrm{Co},{ }^{60} \mathrm{Co},{ }^{88} \mathrm{Y},{ }^{113} \mathrm{Sn},{ }^{133} \mathrm{Ba},{ }^{137} \mathrm{Cs},{ }^{139} \mathrm{Ce},{ }^{152} \mathrm{Eu}\right.$, ${ }^{228}$ Th, $\left.{ }^{241} \mathrm{Am}\right)$.

The primary analysis of the measured $\gamma$-ray spectra was performed using the DEIMOS32 code [20]. The program allows determining the areas under the peaks and their positions (channel number). After that, using a software package [21], the spectra were calibrated for energy, corrected for the detector efficiency, and separate $\gamma$-ray lines of the product nuclei were identified as formed in the samples as a result of interactions with secondary neutrons, protons or deuterons. Experimental count rates of the individual $\gamma$-ray transitions were corrected for the nuclear decay during the irradiation as well as for the self-absorption for the $\gamma$-rays measured, for the geometric dimensions of the samples, for the true coincidence summing [22], for the beam interruptions during irradiation and the variations in intensity of the deuteron beam (based on the on-line measurements with fast ionization chambers). All these procedures are described in detail in Refs. [21, 23, 24].

For determining the experimental reaction rates, the following equation was used [24]:

$$
R\left(A_{r}, Z_{r}\right)=\frac{Q\left(A_{r}, Z_{r}\right)}{N_{t} N_{d}},
$$

where $Q\left(A_{r}, Z_{r}\right)$ is the production rate of the radioactive nucleus $\left(A_{r}, Z_{r}\right), N_{t}$ the number of atoms in the sample, and $N_{d}$ the number of incident deuterons on the target.

\section{MONTE CARLO SIMULATIONS}

The FLUKA hadron-nucleon interaction models are based on resonance production and decay at energies below a few $\mathrm{GeV}$, and on the Dual Parton model at energies above. Two models are also used in hadron-nucleus interactions. At momenta below 3-5 GeV/c, the PEANUT package $[25,26]$ includes a very detailed Generalised Intra-Nuclear Cascade (GINC) and a preequilibrium stage, while at high energies the Gribov-Glauber multiple collision mechanism is included in a less refined GINC. Both modules are followed by equilibrium processes: evaporation, fission, Fermi break-up, $\gamma$-ray de-excitation [27,28]. Inelastic cross sections for hadron-hadron interactions are represented by parameterised fits 

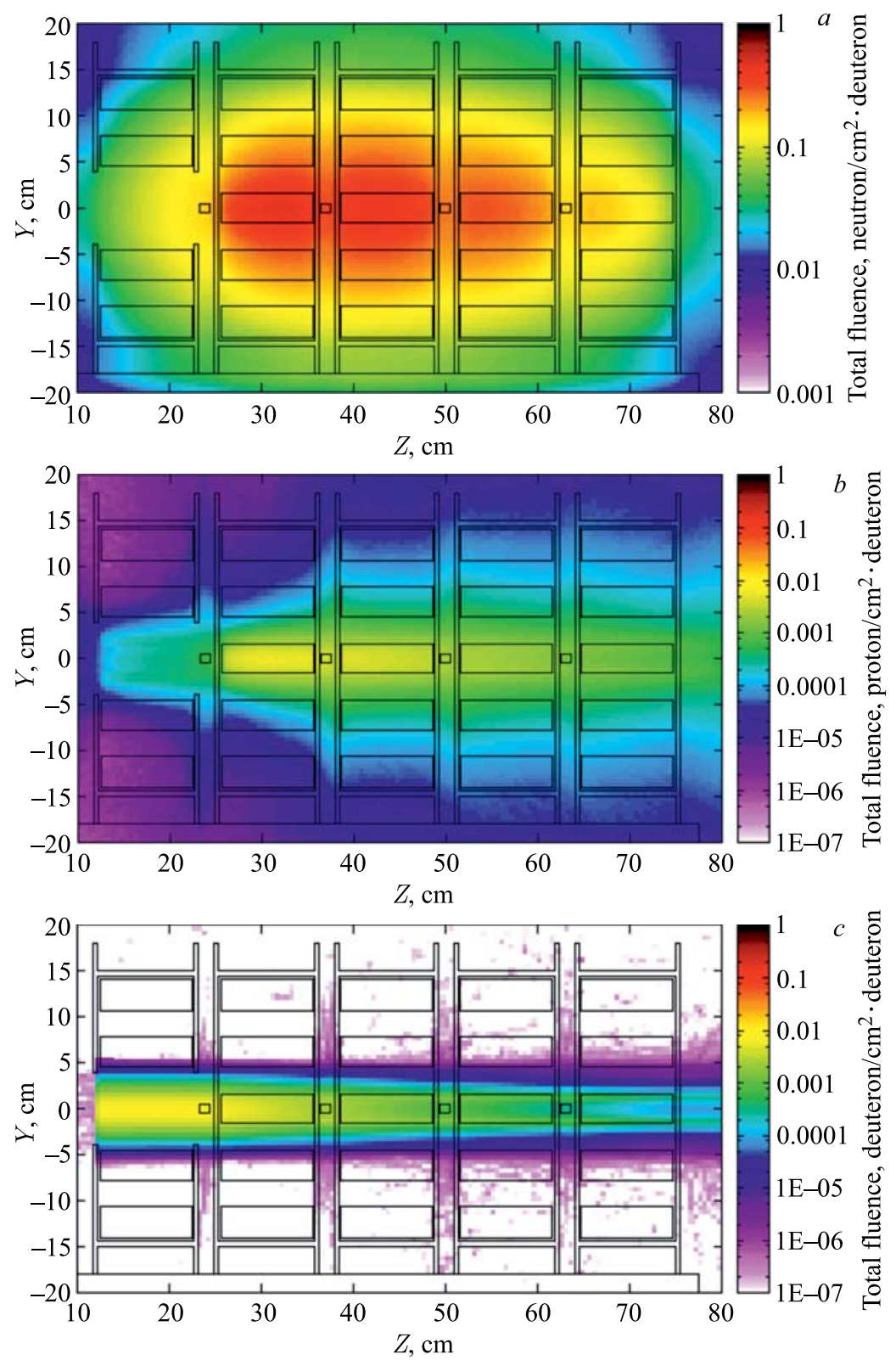

Fig. 2. Total fluence of secondary neutrons $(a)$, total fluence of secondary protons $(b)$ and total fluence of primary and secondary deuterons in the setup QUINTA $(c)$. Shown is side view of the setup geometry cut from the center by $Z$ axis 
based on available experimental data [41]. For hadron-nucleus interactions, a mixture of tabulated data and parameterised fits is used [29-33]. Elastic and charge-exchange reactions are described by phase-shift analyses and eikonal approximation. Ion-induced nuclear interactions are described for energy between 0.1 and $5 \mathrm{GeV} /$ nucleon with modified Relativistic Quantum Molecular Dynamics (RQMD) model [34-36] and for energy below $0.1 \mathrm{GeV} /$ nucleon with Boltzmann Master Equation (BME) theory [37-39].

Figure 2 shows $(a)$ the total fluence of secondary neutrons, $(b)$ the total fluence of secondary protons and $(c)$ the total fluence of primary and secondary deuterons in the QUINTA setup (cut geometry from the center by $Z$ axis is shown conventionally). By $Y$ and $Z$ axes the size of the setup is shown. Fluence was produced by one $6-\mathrm{GeV}$ deuteron and averaged by the $X$ axis. A calculated double differential fluence of secondary neutrons for the position of the thorium samples is presented in Fig. 3. As can be seen from the picture, the biggest fluence of neutrons is for sample 10Th and the smallest fluence is for samples 9Th and $12 \mathrm{Th}$ at energies below $10 \mathrm{MeV}$ and at energies above $10 \mathrm{MeV}$ smallest fluence is only for sample 9Th. The deuteron beam parameters used in the simulations were determined experimentally in Ref. [5]. Coordinates of the center of the beam are $X_{\mathrm{C}}=2.0 \mathrm{~cm}, Y_{\mathrm{C}}=-0.1 \mathrm{~cm}$ and FWHM-distribution is $\mathrm{FWHM}_{X}=3.9 \mathrm{~cm}$, $\mathrm{FWHM}_{Y}=3.1 \mathrm{~cm}$. The dependence of reaction rate on mass of the residual isotopes for the samples 9Th, 10Th, 11Th and 12Th is shown in Fig. 4, in units of nuclei $/ \mathrm{cm}^{3} \cdot$ deuteron. The products of evaporation, fragmentation, fission (with low and high energy particles), spallation and quasi-elastic reactions in the thorium samples can be seen in Fig. 4.

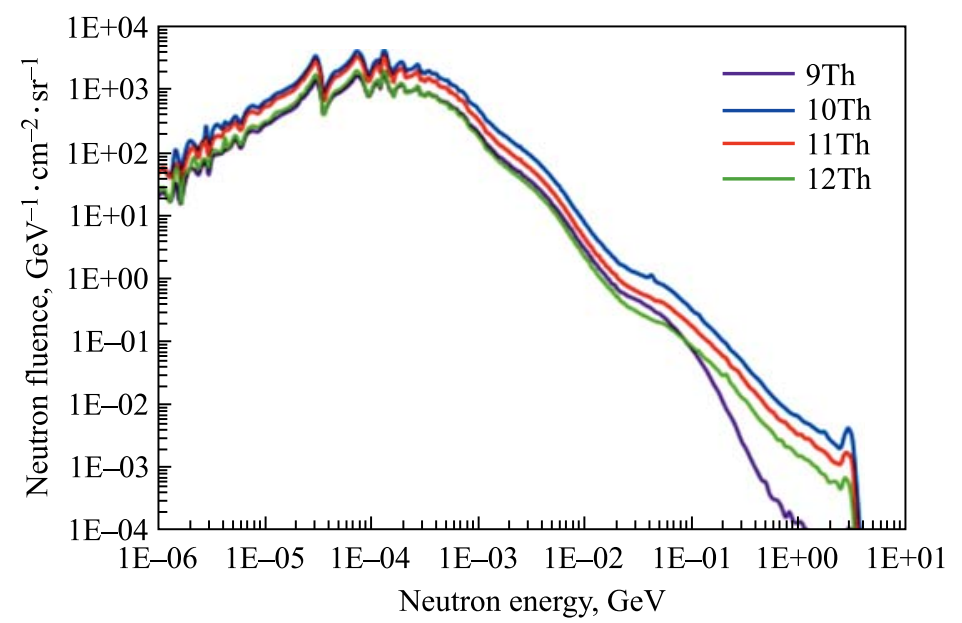

Fig. 3. Double differential fluence of secondary neutrons for the position of the samples 9Th, 10Th, 11Th and $12 \mathrm{Th}$ 


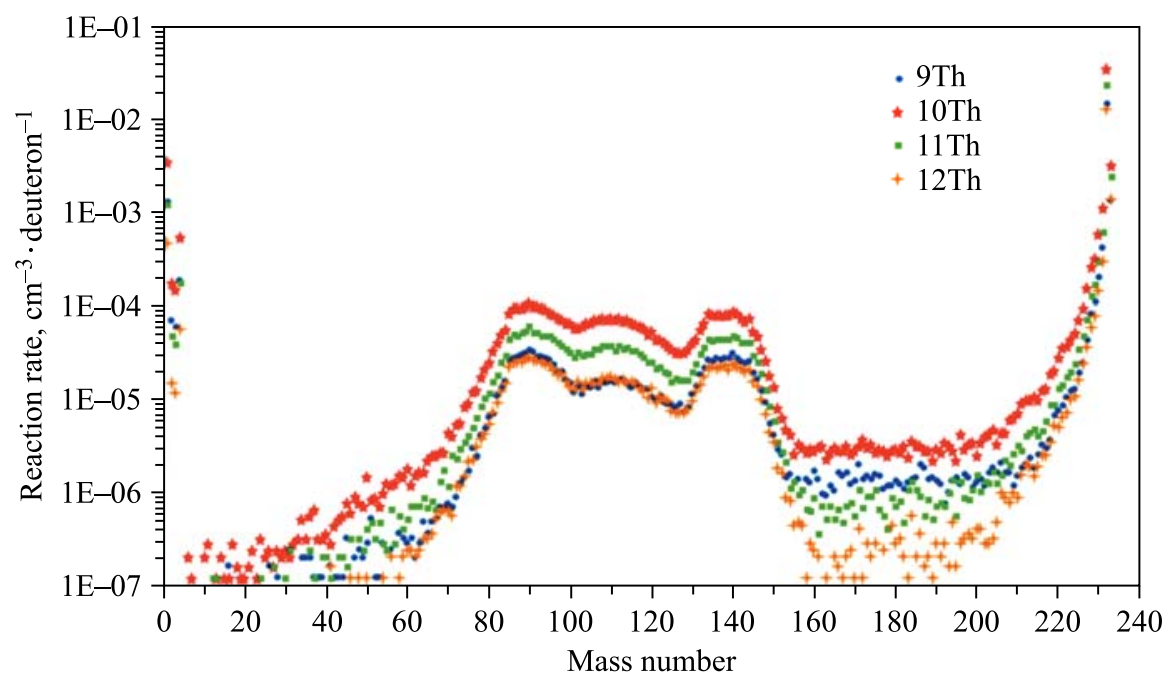

Fig. 4. Dependence of reaction rate on mass of the residual isotopes for the samples 9Th, $10 \mathrm{Th}, 11 \mathrm{Th}$ and $12 \mathrm{Th}$

In the results of simulations, detailed information is taken about the number of fissions in each of the natural uranium bars of the QUINTA setup. In Table 1 the number of fissions for natural uranium bars per one $6-\mathrm{GeV}$ deuteron is given and bars are marked as shown in Fig. 5. The total number of fissions in the setup generated by one $6-\mathrm{GeV}$ deuteron is 51.3 , and 14.9 fissions of them are generated with high-energy $(E>20 \mathrm{MeV})$ particles. From the fissions with high-energy $(E>20 \mathrm{MeV})$ particles, 0.34 fissions are generated by deuterons, 1.7 by protons, 11.3 by neutrons, 0.37 by positive pions, and 1.22 by negative pions. The total number of fissions in the 1 st section is 2.7 , in the 2 nd section is 16.4 , in the 3rd section is 16.0 , in the 4 th section is 10.7 , and in the 5 th section is 5.6. The number of fissions with high-energy $(E>20 \mathrm{MeV})$ particles in the 1st section is 0.52 , in the 2 nd section is 4.96 , in the 3 rd section is 4.66 , in the 4 th section is 3.08 , and in the 5 th section is 1.72 .

Table 1. Number of fissions for natural uranium bars per one 6-GeV deuteron

\begin{tabular}{|c|c|c|c|c|c|}
\hline $\begin{array}{c}\text { Number } \\
\text { of bars }\end{array}$ & 1st section & 2nd section & 3rd section & 4th section & 5th section \\
\hline U01 & $1.94 \mathrm{E}-02$ & $4.43 \mathrm{E}-02$ & $5.63 \mathrm{E}-02$ & $4.38 \mathrm{E}-02$ & $2.49 \mathrm{E}-02$ \\
\hline $\mathrm{U} 02$ & $2.56 \mathrm{E}-02$ & $6.40 \mathrm{E}-02$ & $8.09 \mathrm{E}-02$ & $6.19 \mathrm{E}-02$ & $3.50 \mathrm{E}-02$ \\
\hline $\mathrm{U} 03$ & $3.06 \mathrm{E}-02$ & $8.21 \mathrm{E}-02$ & $1.03 \mathrm{E}-01$ & $7.88 \mathrm{E}-02$ & $4.46 \mathrm{E}-02$ \\
\hline U04 & $3.18 \mathrm{E}-02$ & $8.73 \mathrm{E}-02$ & $1.12 \mathrm{E}-01$ & $8.68 \mathrm{E}-02$ & $4.98 \mathrm{E}-02$ \\
\hline U05 & $2.75 \mathrm{E}-02$ & $7.46 \mathrm{E}-02$ & $9.84 \mathrm{E}-02$ & $7.84 \mathrm{E}-02$ & $4.63 \mathrm{E}-02$ \\
\hline
\end{tabular}


Table 1 continued

\begin{tabular}{|c|c|c|c|c|c|}
\hline $\begin{array}{l}\text { Number } \\
\text { of bars }\end{array}$ & 1st section & 2nd section & 3rd section & 4th section & 5th section \\
\hline U06 & $2.30 \mathrm{E}-02$ & $5.26 \mathrm{E}-02$ & $6.54 \mathrm{E}-02$ & $4.96 \mathrm{E}-02$ & $2.79 \mathrm{E}-02$ \\
\hline U07 & $3.42 \mathrm{E}-02$ & $8.81 \mathrm{E}-02$ & $1.07 \mathrm{E}-01$ & $7.92 \mathrm{E}-02$ & $4.38 \mathrm{E}-02$ \\
\hline U08 & 4.39E-02 & $1.30 \mathrm{E}-01$ & $1.54 \mathrm{E}-01$ & $1.12 \mathrm{E}-01$ & $6.15 \mathrm{E}-02$ \\
\hline U09 & $5.09 \mathrm{E}-02$ & $1.65 \mathrm{E}-01$ & $1.97 \mathrm{E}-01$ & $1.43 \mathrm{E}-01$ & $7.79 \mathrm{E}-02$ \\
\hline U10 & $4.85 \mathrm{E}-02$ & $1.58 \mathrm{E}-01$ & $1.97 \mathrm{E}-01$ & $1.48 \mathrm{E}-01$ & $8.44 \mathrm{E}-02$ \\
\hline U11 & $3.72 \mathrm{E}-02$ & $1.12 \mathrm{E}-01$ & $1.47 \mathrm{E}-01$ & $1.16 \mathrm{E}-01$ & $6.90 \mathrm{E}-02$ \\
\hline U12 & $2.33 \mathrm{E}-02$ & $5.30 \mathrm{E}-02$ & $6.56 \mathrm{E}-02$ & 4.94E-02 & $2.76 \mathrm{E}-02$ \\
\hline U13 & 4.01E-02 & $9.87 \mathrm{E}-02$ & $1.16 \mathrm{E}-01$ & 8.42E-02 & 4.59E-02 \\
\hline U14 & $6.23 \mathrm{E}-02$ & $1.76 \mathrm{E}-01$ & $1.96 \mathrm{E}-01$ & $1.35 \mathrm{E}-01$ & $7.12 \mathrm{E}-02$ \\
\hline U15 & $7.79 \mathrm{E}-02$ & $2.90 \mathrm{E}-01$ & $3.06 \mathrm{E}-01$ & $2.02 \mathrm{E}-01$ & $1.05 \mathrm{E}-01$ \\
\hline U16 & $8.35 \mathrm{E}-02$ & $3.54 \mathrm{E}-01$ & $3.86 \mathrm{E}-01$ & $2.63 \mathrm{E}-01$ & $1.39 \mathrm{E}-01$ \\
\hline U17 & $6.82 \mathrm{E}-02$ & $2.57 \mathrm{E}-01$ & $3.13 \mathrm{E}-01$ & $2.34 \mathrm{E}-01$ & $1.33 \mathrm{E}-01$ \\
\hline U18 & $4.49 \mathrm{E}-02$ & $1.45 \mathrm{E}-01$ & $1.91 \mathrm{E}-01$ & $1.52 \mathrm{E}-01$ & $9.09 \mathrm{E}-02$ \\
\hline U19 & $2.08 \mathrm{E}-02$ & $4.58 \mathrm{E}-02$ & $5.65 \mathrm{E}-02$ & 4.29E-02 & $2.41 \mathrm{E}-02$ \\
\hline U20 & $3.66 \mathrm{E}-02$ & $9.08 \mathrm{E}-02$ & $1.06 \mathrm{E}-01$ & $7.68 \mathrm{E}-02$ & $4.16 \mathrm{E}-02$ \\
\hline $\mathrm{U} 21$ & $6.47 \mathrm{E}-02$ & $1.82 \mathrm{E}-01$ & $1.95 \mathrm{E}-01$ & $1.31 \mathrm{E}-01$ & $6.86 \mathrm{E}-02$ \\
\hline $\mathrm{U} 22$ & & $4.22 \mathrm{E}-01$ & $3.79 \mathrm{E}-01$ & $2.32 \mathrm{E}-01$ & $1.14 \mathrm{E}-01$ \\
\hline $\mathrm{U} 23$ & & $1.02 \mathrm{E}+00$ & $7.84 \mathrm{E}-01$ & 4.33E-01 & $1.99 \mathrm{E}-01$ \\
\hline $\mathrm{U} 24$ & $1.79 \mathrm{E}-01$ & $8.10 \mathrm{E}-01$ & $8.28 \mathrm{E}-01$ & $5.51 \mathrm{E}-01$ & $2.83 \mathrm{E}-01$ \\
\hline $\mathrm{U} 25$ & $8.23 \mathrm{E}-02$ & $3.30 \mathrm{E}-01$ & $4.06 \mathrm{E}-01$ & $3.14 \mathrm{E}-01$ & $1.87 \mathrm{E}-01$ \\
\hline $\mathrm{U} 26$ & 4.42E-02 & $1.43 \mathrm{E}-01$ & $1.91 \mathrm{E}-01$ & $1.56 \mathrm{E}-01$ & $9.64 \mathrm{E}-02$ \\
\hline U27 & $1.59 \mathrm{E}-02$ & $3.34 \mathrm{E}-02$ & $4.17 \mathrm{E}-02$ & $3.25 \mathrm{E}-02$ & $1.87 \mathrm{E}-02$ \\
\hline U28 & $2.96 \mathrm{E}-02$ & $6.82 \mathrm{E}-02$ & $8.23 \mathrm{E}-02$ & $6.10 \mathrm{E}-02$ & $3.37 \mathrm{E}-02$ \\
\hline U29 & $5.54 \mathrm{E}-02$ & $1.36 \mathrm{E}-01$ & $1.52 \mathrm{E}-01$ & $1.05 \mathrm{E}-01$ & $5.59 \mathrm{E}-02$ \\
\hline U30 & & $3.19 \mathrm{E}-01$ & $2.97 \mathrm{E}-01$ & $1.86 \mathrm{E}-01$ & $9.31 \mathrm{E}-02$ \\
\hline $\mathrm{U} 31$ & & $1.29 \mathrm{E}+00$ & $7.75 \mathrm{E}-01$ & $3.87 \mathrm{E}-01$ & $1.72 \mathrm{E}-01$ \\
\hline $\mathrm{U} 32$ & & $2.71 \mathrm{E}+00$ & $1.81 \mathrm{E}+00$ & $8.96 \mathrm{E}-01$ & $3.68 \mathrm{E}-01$ \\
\hline U33 & $1.74 \mathrm{E}-01$ & $7.95 \mathrm{E}-01$ & $9.03 \mathrm{E}-01$ & $6.56 \mathrm{E}-01$ & $3.62 \mathrm{E}-01$ \\
\hline U34 & $6.66 \mathrm{E}-02$ & $2.46 \mathrm{E}-01$ & $3.19 \mathrm{E}-01$ & $2.56 \mathrm{E}-01$ & $1.58 \mathrm{E}-01$ \\
\hline U35 & $3.36 \mathrm{E}-02$ & $1.02 \mathrm{E}-01$ & $1.40 \mathrm{E}-01$ & $1.18 \mathrm{E}-01$ & $7.37 \mathrm{E}-02$ \\
\hline U36 & $2.04 \mathrm{E}-02$ & $4.52 \mathrm{E}-02$ & $5.58 \mathrm{E}-02$ & $4.26 \mathrm{E}-02$ & $2.38 \mathrm{E}-02$ \\
\hline U37 & $3.63 \mathrm{E}-02$ & 8.92E-02 & $1.05 \mathrm{E}-01$ & $7.62 \mathrm{E}-02$ & $4.14 \mathrm{E}-02$ \\
\hline U38 & $6.39 \mathrm{E}-02$ & $1.79 \mathrm{E}-01$ & $1.91 \mathrm{E}-01$ & $1.30 \mathrm{E}-01$ & $6.73 \mathrm{E}-02$ \\
\hline U39 & & $4.06 \mathrm{E}-01$ & $3.70 \mathrm{E}-01$ & $2.27 \mathrm{E}-01$ & $1.12 \mathrm{E}-01$ \\
\hline $\mathrm{U} 40$ & & $9.38 \mathrm{E}-01$ & $7.39 \mathrm{E}-01$ & $4.16 \mathrm{E}-01$ & $1.91 \mathrm{E}-01$ \\
\hline $\mathrm{U} 41$ & $1.61 \mathrm{E}-01$ & $7.59 \mathrm{E}-01$ & $7.80 \mathrm{E}-01$ & $5.21 \mathrm{E}-01$ & $2.67 \mathrm{E}-01$ \\
\hline U42 & $7.98 \mathrm{E}-02$ & $3.22 \mathrm{E}-01$ & $3.97 \mathrm{E}-01$ & $3.06 \mathrm{E}-01$ & $1.82 \mathrm{E}-01$ \\
\hline
\end{tabular}


Table 1 continued

\begin{tabular}{|c|c|c|c|c|c|}
\hline $\begin{array}{c}\text { Number } \\
\text { of bars }\end{array}$ & 1 st section & 2nd section & 3rd section & 4th section & 5th section \\
\hline U43 & $4.31 \mathrm{E}-02$ & $1.40 \mathrm{E}-01$ & $1.88 \mathrm{E}-01$ & $1.54 \mathrm{E}-01$ & $9.51 \mathrm{E}-02$ \\
\hline U44 & $2.29 \mathrm{E}-02$ & $5.16 \mathrm{E}-02$ & $6.37 \mathrm{E}-02$ & $4.81 \mathrm{E}-02$ & $2.70 \mathrm{E}-02$ \\
\hline U45 & $3.93 \mathrm{E}-02$ & $9.68 \mathrm{E}-02$ & $1.13 \mathrm{E}-01$ & $8.25 \mathrm{E}-02$ & $4.46 \mathrm{E}-02$ \\
\hline U46 & $6.11 \mathrm{E}-02$ & $1.71 \mathrm{E}-01$ & $1.90 \mathrm{E}-01$ & $1.32 \mathrm{E}-01$ & $6.95 \mathrm{E}-02$ \\
\hline U47 & $7.62 \mathrm{E}-02$ & $2.76 \mathrm{E}-01$ & $2.94 \mathrm{E}-01$ & $1.96 \mathrm{E}-01$ & $1.01 \mathrm{E}-01$ \\
\hline U48 & $8.05 \mathrm{E}-02$ & $3.35 \mathrm{E}-01$ & $3.68 \mathrm{E}-01$ & $2.52 \mathrm{E}-01$ & $1.34 \mathrm{E}-01$ \\
\hline U49 & $6.51 \mathrm{E}-02$ & $2.46 \mathrm{E}-01$ & $3.02 \mathrm{E}-01$ & $2.26 \mathrm{E}-01$ & $1.29 \mathrm{E}-01$ \\
\hline U50 & $4.32 \mathrm{E}-02$ & $1.40 \mathrm{E}-01$ & $1.85 \mathrm{E}-01$ & $1.48 \mathrm{E}-01$ & $8.87 \mathrm{E}-02$ \\
\hline U51 & $2.20 \mathrm{E}-02$ & $5.02 \mathrm{E}-02$ & $6.29 \mathrm{E}-02$ & $4.79 \mathrm{E}-02$ & $2.70 \mathrm{E}-02$ \\
\hline U52 & $3.30 \mathrm{E}-02$ & $8.46 \mathrm{E}-02$ & $1.03 \mathrm{E}-01$ & $7.66 \mathrm{E}-02$ & $4.25 \mathrm{E}-02$ \\
\hline U53 & $4.23 \mathrm{E}-02$ & $1.24 \mathrm{E}-01$ & $1.48 \mathrm{E}-01$ & $1.08 \mathrm{E}-01$ & $5.89 \mathrm{E}-02$ \\
\hline U54 & $4.88 \mathrm{E}-02$ & $1.57 \mathrm{E}-01$ & $1.88 \mathrm{E}-01$ & $1.37 \mathrm{E}-01$ & $7.46 \mathrm{E}-02$ \\
\hline U55 & $4.63 \mathrm{E}-02$ & $1.50 \mathrm{E}-01$ & $1.88 \mathrm{E}-01$ & $1.42 \mathrm{E}-01$ & $8.08 \mathrm{E}-02$ \\
\hline U56 & $3.53 \mathrm{E}-02$ & $1.07 \mathrm{E}-01$ & $1.40 \mathrm{E}-01$ & $1.12 \mathrm{E}-01$ & $6.61 \mathrm{E}-02$ \\
\hline U57 & $1.80 \mathrm{E}-02$ & $4.11 \mathrm{E}-02$ & $5.21 \mathrm{E}-02$ & $4.07 \mathrm{E}-02$ & $2.33 \mathrm{E}-02$ \\
\hline U58 & $2.39 \mathrm{E}-02$ & $5.99 \mathrm{E}-02$ & $7.53 \mathrm{E}-02$ & $5.80 \mathrm{E}-02$ & $3.32 \mathrm{E}-02$ \\
\hline U59 & $2.86 \mathrm{E}-02$ & $7.63 \mathrm{E}-02$ & $9.68 \mathrm{E}-02$ & $7.38 \mathrm{E}-02$ & $4.18 \mathrm{E}-02$ \\
\hline U60 & $2.94 \mathrm{E}-02$ & $8.17 \mathrm{E}-02$ & $1.05 \mathrm{E}-01$ & $8.15 \mathrm{E}-02$ & $4.65 \mathrm{E}-02$ \\
\hline U61 & $2.56 \mathrm{E}-02$ & $6.94 \mathrm{E}-02$ & $9.23 \mathrm{E}-02$ & $7.38 \mathrm{E}-02$ & $4.34 \mathrm{E}-02$ \\
\hline
\end{tabular}

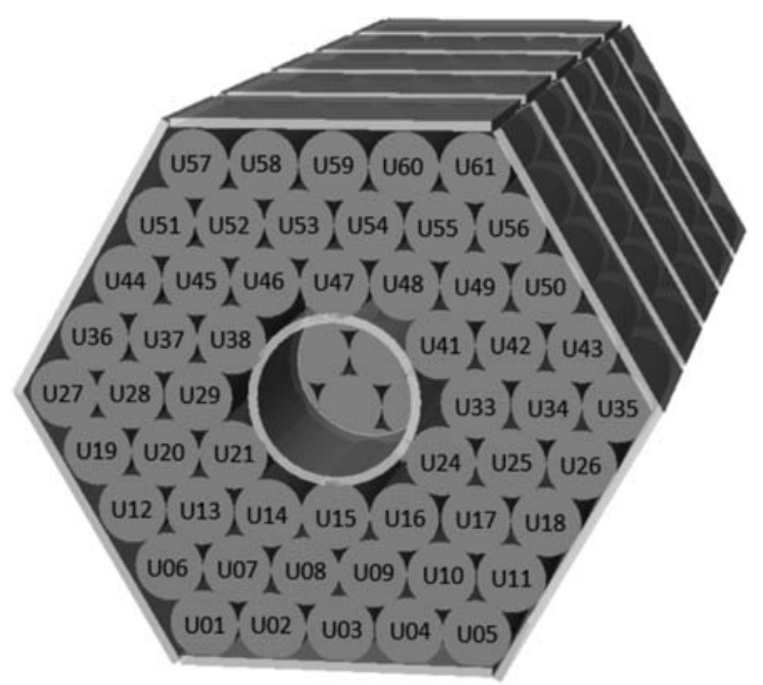

Fig. 5. Marking of nat $U$ bars of the QUINTA setup for the definition of fission numbers 


\section{EXPERIMENTAL RESULTS AND COMPARISONS WITH MONTE CARLO SIMULATIONS}

Table 2 gives the cumulative reaction rates for residual radionuclides obtained from the experiment. As can be seen from the table, for the sample 10Th the biggest values of reaction rates are for almost all residual nuclei and the smallest values of reaction rates are for sample $12 \mathrm{Th} .{ }^{233} \mathrm{~Pa}$ is produced in the reaction ${ }^{232} \mathrm{Th}(n, \gamma){ }^{233} \mathrm{Th}\left(\beta^{-}\right.$decay, $\left.T_{1 / 2}=22.3 \mathrm{~min}\right) \rightarrow{ }^{233} \mathrm{~Pa}\left(\beta^{-}\right.$decay, $T_{1 / 2}=$ 26.967 day $\rightarrow{ }^{233} \mathrm{U}$. Ratio of the weight of produced ${ }^{233} \mathrm{U}$ to ${ }^{232} \mathrm{Th}$ for the sample 9 Th is $1.39(4) \mathrm{E}-12$, for $10 \mathrm{Th}$ is $3.51(10) \mathrm{E}-12$, for $11 \mathrm{Th}$ is $2.39(8) \mathrm{E}-12$, and for $12 \mathrm{Th}$ equals $1.48(4) \mathrm{E}-12$.

Table 2. Experimentally obtained cumulative reaction rates for residual radionuclides

\begin{tabular}{|c|c|c|c|c|}
\hline \multirow{2}{*}{$\begin{array}{c}\text { Residual } \\
\text { nuclei }\end{array}$} & \multicolumn{4}{|c|}{ Reaction rate $\left(\right.$ atom $^{-1} \cdot$ deuteron $\left.^{-1}\right)$} \\
\hline & 9Th & $10 \mathrm{Th}$ & $11 \mathrm{Th}$ & $12 \mathrm{Th}$ \\
\hline $\mathrm{Be}-7$ & & $9.0(11) \mathrm{E}-27$ & & \\
\hline $\mathrm{Na}-22$ & & $7.5(16) \mathrm{E}-27$ & & \\
\hline $\mathrm{Na}-24$ & $1.47(5) \mathrm{E}-27$ & $1.00(2) \mathrm{E}-27$ & $3.78(22) \mathrm{E}-28$ & $1.25(18) \mathrm{E}-28$ \\
\hline $\mathrm{Mg}-28$ & $6.44(48) \mathrm{E}-28$ & & & \\
\hline $\mathrm{S}-38$ & $2.73(61) \mathrm{E}-28$ & & & \\
\hline Ar-41 & & & $5.00(71) \mathrm{E}-28$ & \\
\hline $\mathrm{K}-42$ & $6.11(95) \mathrm{E}-28$ & & & \\
\hline $\mathrm{K}-43$ & $2.09(9) \mathrm{E}-27$ & $4.01(5) \mathrm{E}-28$ & $2.84(25) \mathrm{E}-28$ & $1.08(4) \mathrm{E}-27$ \\
\hline Sc-43 & & $7.8(14) \mathrm{E}-28$ & & \\
\hline $\mathrm{Ca}-47$ & & $2.07(31) \mathrm{E}-28$ & & \\
\hline Sc-47 & & $5.51(52) \mathrm{E}-28$ & & \\
\hline Sc-48 & $3.71(25) \mathrm{E}-28$ & & & \\
\hline V-48 & & $1.05(18) \mathrm{E}-27$ & & \\
\hline Mn-52 & & $1.90(49) \mathrm{E}-27$ & & \\
\hline Mn-56 & $1.33(25) \mathrm{E}-27$ & & & \\
\hline $\mathrm{Ni}-56$ & & $7.8(36) \mathrm{E}-28$ & & \\
\hline $\mathrm{Fe}-59$ & & $7.5(11) \mathrm{E}-28$ & & \\
\hline Ga-66 & $9.7(27) \mathrm{E}-28$ & $1.22(22) \mathrm{E}-27$ & & $3.48(43) \mathrm{E}-28$ \\
\hline Ge-66 & & & & $1.57(28) \mathrm{E}-27$ \\
\hline $\mathrm{Zn}-69 \mathrm{~m}$ & $3.07(18) \mathrm{E}-28$ & $3.03(16) \mathrm{E}-28$ & $2.22(15) \mathrm{E}-28$ & $7.9(11) \mathrm{E}-29$ \\
\hline As-70 & & $1.50(27) \mathrm{E}-27$ & $1.65(29) \mathrm{E}-27$ & \\
\hline $\mathrm{Zn}-71 \mathrm{~m}$ & & $2.39(24) \mathrm{E}-28$ & & \\
\hline As-71 & $1.97(19) \mathrm{E}-28$ & & & \\
\hline As-72 & & $1.84(70) \mathrm{E}-27$ & $6.2(11) \mathrm{E}-28$ & \\
\hline
\end{tabular}


Table 2 continued

\begin{tabular}{|c|c|c|c|c|}
\hline \multirow{2}{*}{$\begin{array}{c}\text { Residual } \\
\text { nuclei }\end{array}$} & \multicolumn{4}{|c|}{ Reaction rate $\left(\right.$ atom $^{-1} \cdot$ deuteron $\left.^{-1}\right)$} \\
\hline & 9Th & $10 \mathrm{Th}$ & $11 \mathrm{Th}$ & $12 \mathrm{Th}$ \\
\hline Ga-72 & & $8.8(19) \mathrm{E}-28$ & $1.02(13) \mathrm{E}-28$ & \\
\hline Ga-73 & & $1.12(19) \mathrm{E}-27$ & & \\
\hline Se-73 & & $1.87(29) \mathrm{E}-28$ & & \\
\hline As-74 & $7.3(10) \mathrm{E}-28$ & $2.10(36) \mathrm{E}-27$ & & \\
\hline As-76 & & $9.77(68) \mathrm{E}-28$ & & \\
\hline Br-76 & & $6.17(96) \mathrm{E}-28$ & $2.97(27) \mathrm{E}-28$ & \\
\hline Ge-77 & & $1.01(14) \mathrm{E}-27$ & $4.46(62) \mathrm{E}-28$ & $1.43(33) \mathrm{E}-28$ \\
\hline As-78 & $7.8(13) \mathrm{E}-28$ & & & \\
\hline Rb-81 & & $3.91(29) \mathrm{E}-28$ & & \\
\hline Br-82 & & $9.61(34) \mathrm{E}-28$ & $6.78(65) \mathrm{E}-28$ & \\
\hline Rb-82m & & $6.87(63) \mathrm{E}-28$ & & \\
\hline Rb-83 & $2.95(35) \mathrm{E}-27$ & $1.47(30) \mathrm{E}-27$ & & \\
\hline Sr-85 & $1.45(12) \mathrm{E}-27$ & & & \\
\hline Kr-85m & $2.09(8) \mathrm{E}-27$ & $3.92(61) \mathrm{E}-27$ & $3.01(28) \mathrm{E}-27$ & $1.30(9) \mathrm{E}-27$ \\
\hline Y-85m & & $6.1(19) \mathrm{E}-28$ & & \\
\hline Rb-86 & & $1.12(37) \mathrm{E}-27$ & & \\
\hline Y-86 & & $4.08(42) \mathrm{E}-28$ & & \\
\hline Zr-86 & $4.80(28) \mathrm{E}-28$ & $4.48(44) \mathrm{E}-28$ & $2.42(16) \mathrm{E}-28$ & \\
\hline Kr-87 & & & $3.08(32) \mathrm{E}-27$ & $1.29(12) \mathrm{E}-27$ \\
\hline Y-87 & & & $4.66(89) \mathrm{E}-28$ & \\
\hline Y-87m & $1.22(9) \mathrm{E}-27$ & $1.07(5) \mathrm{E}-27$ & $5.43(63) \mathrm{E}-28$ & $2.55(20) \mathrm{E}-28$ \\
\hline Sr-87m & & $1.28(38) \mathrm{E}-28$ & & \\
\hline Kr-88 & $2.64(57) \mathrm{E}-27$ & $4.60(18) \mathrm{E}-27$ & $3.04(29) \mathrm{E}-27$ & $1.67(17) \mathrm{E}-27$ \\
\hline Y-88 & & $1.45(18) \mathrm{E}-27$ & & \\
\hline Nb-89m & & $4.22(88) \mathrm{E}-28$ & & \\
\hline Nb-90 & $4.02(34) \mathrm{E}-28$ & $1.12(3) \mathrm{E}-27$ & & \\
\hline Y-90m & $1.06(10) \mathrm{E}-27$ & & $6.44(65) \mathrm{E}-28$ & $2.46(17) \mathrm{E}-28$ \\
\hline Y-91m & & $1.29(5) \mathrm{E}-26$ & & \\
\hline Sr-91 & $2.96(17) \mathrm{E}-27$ & $5.90(27) \mathrm{E}-27$ & $4.42(17) \mathrm{E}-27$ & $2.06(11) \mathrm{E}-27$ \\
\hline Sr-92 & $2.86(18) \mathrm{E}-27$ & $5.17(16) \mathrm{E}-27$ & $3.61(21) \mathrm{E}-27$ & $1.80(13) \mathrm{E}-27$ \\
\hline Y-92 & $4.66(38) \mathrm{E}-27$ & $9.66(70) \mathrm{E}-27$ & $6.45(61) \mathrm{E}-27$ & $3.21(82) \mathrm{E}-27$ \\
\hline Y-93 & $4.95(66) \mathrm{E}-27$ & $5.76(40) \mathrm{E}-27$ & $5.41(31) \mathrm{E}-27$ & $2.08(18) \mathrm{E}-27$ \\
\hline Mo-93m & $3.46(58) \mathrm{E}-28$ & $2.72(81) \mathrm{E}-28$ & & \\
\hline Tc-94 & $3.51(46) \mathrm{E}-28$ & & & \\
\hline Tc-94m & & $1.28(19) \mathrm{E}-27$ & & \\
\hline Ru-94 & & $2.21(30) \mathrm{E}-27$ & & \\
\hline
\end{tabular}


Table 2 continued

\begin{tabular}{|c|c|c|c|c|}
\hline \multirow{2}{*}{$\begin{array}{c}\text { Residual } \\
\text { nuclei }\end{array}$} & \multicolumn{4}{|c|}{ Reaction rate $\left(\right.$ atom $^{-1} \cdot$ deuteron $\left.^{-1}\right)$} \\
\hline & 9Th & $10 \mathrm{Th}$ & $11 \mathrm{Th}$ & $12 \mathrm{Th}$ \\
\hline Zr-95 & & $7.47(61) \mathrm{E}-27$ & $4.90(32) \mathrm{E}-27$ & \\
\hline Nb-95 & & $2.87(51) \mathrm{E}-27$ & & \\
\hline Tc-95 & & $5.1(13) \mathrm{E}-28$ & & \\
\hline Ru-95 & & $3.66(27) \mathrm{E}-27$ & & \\
\hline Nb-96 & $7.96(96) \mathrm{E}-28$ & $1.39(14) \mathrm{E}-27$ & $8.37(55) \mathrm{E}-28$ & $3.96(40) \mathrm{E}-28$ \\
\hline Tc-96 & & $5.7(10) \mathrm{E}-28$ & & \\
\hline Zr-97 & $2.36(11) \mathrm{E}-27$ & $5.09(16) \mathrm{E}-27$ & $3.41(14) \mathrm{E}-27$ & $1.69(6) \mathrm{E}-27$ \\
\hline Nb-97 & & $4.94(17) \mathrm{E}-27$ & & \\
\hline Nb-98m & & $1.71(22) \mathrm{E}-27$ & & \\
\hline Tc-99m & & $1.21(5) \mathrm{E}-27$ & & \\
\hline Mo-99 & $3.48(37) \mathrm{E}-27$ & & $4.31(30) \mathrm{E}-27$ & $2.04(12) \mathrm{E}-27$ \\
\hline Rh-99 & & $2.83(15) \mathrm{E}-27$ & & \\
\hline Rh-99m & & $4.5(11) \mathrm{E}-28$ & & \\
\hline Rh-100 & $4.54(27) \mathrm{E}-28$ & $4.62(57) \mathrm{E}-28$ & & \\
\hline Rh-101m & $9.87(56) \mathrm{E}-28$ & $7.9(12) \mathrm{E}-28$ & & \\
\hline Pd-101 & & $1.40(16) \mathrm{E}-27$ & & \\
\hline Ru-103 & $3.12(14) \mathrm{E}-27$ & $7.35(42) \mathrm{E}-27$ & $4.11(17) \mathrm{E}-27$ & $1.94(12) \mathrm{E}-27$ \\
\hline Ru-105 & $2.95(13) \mathrm{E}-27$ & $5.16(11) \mathrm{E}-27$ & $3.65(14) \mathrm{E}-27$ & $1.57(13) \mathrm{E}-27$ \\
\hline Rh-105 & $2.84(21) \mathrm{E}-27$ & $5.89(23) \mathrm{E}-27$ & $3.82(28) \mathrm{E}-27$ & $1.80(12) \mathrm{E}-27$ \\
\hline In-108 & & $9.0(24) \mathrm{E}-28$ & & \\
\hline Ag-110m & & $6.2(33) \mathrm{E}-27$ & $2.65(28) \mathrm{E}-27$ & \\
\hline In-110 & & & $1.70(16) \mathrm{E}-27$ & \\
\hline Pd-111m & & $1.42(6) \mathrm{E}-27$ & $9.2(15) \mathrm{E}-28$ & \\
\hline Ag-111 & & $5.76(72) \mathrm{E}-27$ & & \\
\hline Cd-111m & & $3.91(28) \mathrm{E}-27$ & & \\
\hline In-111 & $8.3(12) \mathrm{E}-28$ & $6.23(22) \mathrm{E}-28$ & $2.67(22) \mathrm{E}-28$ & $1.48(43) \mathrm{E}-28$ \\
\hline Ag-112 & & $6.3(19) \mathrm{E}-27$ & & \\
\hline Ag-113 & & $6.28(71) \mathrm{E}-27$ & $4.93(54) \mathrm{E}-27$ & $2.26(23) \mathrm{E}-27$ \\
\hline Sn-113 & & $1.46(34) \mathrm{E}-27$ & & \\
\hline Cd-115 & $1.55(7) \mathrm{E}-27$ & & $2.44(16) \mathrm{E}-27$ & $1.17(7) \mathrm{E}-27$ \\
\hline In-115m & & $1.24(39) \mathrm{E}-27$ & & \\
\hline In-116m & & $3.94(54) \mathrm{E}-27$ & & \\
\hline Sb-116 & & $6.76(86) \mathrm{E}-28$ & & \\
\hline Sb-116m & & & $1.20(15) \mathrm{E}-27$ & \\
\hline Te-116 & & & & \\
\hline Cd-117 & & & \\
\hline
\end{tabular}


Table 2 continued

\begin{tabular}{|c|c|c|c|c|}
\hline \multirow{2}{*}{$\begin{array}{c}\text { Residual } \\
\text { nuclei }\end{array}$} & \multicolumn{4}{|c|}{ Reaction rate $\left(\right.$ atom $^{-1} \cdot$ deuteron $\left.^{-1}\right)$} \\
\hline & 9Th & $10 \mathrm{Th}$ & $11 \mathrm{Th}$ & $12 \mathrm{Th}$ \\
\hline Cd-117m & & $1.56(11) \mathrm{E}-27$ & $1.23(15) \mathrm{E}-27$ & \\
\hline Sb-117 & $3.65(32) \mathrm{E}-27$ & $6.6(11) \mathrm{E}-27$ & $3.42(21) \mathrm{E}-27$ & \\
\hline Sn-117m & $1.03(18) \mathrm{E}-27$ & & & \\
\hline Sb-118m & $3.17(30) \mathrm{E}-27$ & $4.50(99) \mathrm{E}-28$ & $2.19(25) \mathrm{E}-28$ & $1.47(24) \mathrm{E}-28$ \\
\hline Te-119 & $4.42(61) \mathrm{E}-28$ & $3.00(24) \mathrm{E}-28$ & $2.11(20) \mathrm{E}-28$ & \\
\hline Te-119m & & $3.04(38) \mathrm{E}-28$ & & \\
\hline Sb-120m & & $6.13(69) \mathrm{E}-28$ & $4.49(62) \mathrm{E}-28$ & $1.78(30) \mathrm{E}-28$ \\
\hline $\mathrm{I}-120 \mathrm{~m}$ & & $2.26(72) \mathrm{E}-27$ & & \\
\hline Te-121 & & $7.04(90) \mathrm{E}-28$ & & \\
\hline Te-121m & & $3.03(36) \mathrm{E}-27$ & & \\
\hline $\mathrm{I}-121$ & & $7.44(67) \mathrm{E}-28$ & & \\
\hline Sb-122 & $4.63(70) \mathrm{E}-28$ & $7.88(75) \mathrm{E}-28$ & $5.01(93) \mathrm{E}-28$ & \\
\hline Xe-122 & & $2.40(25) \mathrm{E}-27$ & & \\
\hline Te-123m & & $2.43(91) \mathrm{E}-27$ & & \\
\hline $\mathrm{I}-123$ & & $2.80(28) \mathrm{E}-27$ & & \\
\hline Xe-123 & & $1.09(13) \mathrm{E}-27$ & & \\
\hline Sb-124 & & $1.71(32) \mathrm{E}-27$ & & \\
\hline $\mathrm{I}-124$ & & $7.8(20) \mathrm{E}-28$ & $3.99(40) \mathrm{E}-28$ & \\
\hline $\mathrm{Xe}-125$ & $1.52(9) \mathrm{E}-27$ & $6.78(32) \mathrm{E}-28$ & $3.82(23) \mathrm{E}-28$ & $1.58(21) \mathrm{E}-28$ \\
\hline Sb-126 & & $9.16(44) \mathrm{E}-28$ & & \\
\hline $\mathrm{I}-126$ & & $2.17(22) \mathrm{E}-27$ & & \\
\hline Sb-127 & & $1.16(10) \mathrm{E}-27$ & & \\
\hline Xe-127 & $9.52(91) \mathrm{E}-28$ & $1.16(6) \mathrm{E}-27$ & $8.2(16) \mathrm{E}-28$ & \\
\hline Cs-127 & & $1.50(13) \mathrm{E}-27$ & $7.6(13) \mathrm{E}-27$ & \\
\hline Sb-128 & $3.27(61) \mathrm{E}-28$ & $5.65(53) \mathrm{E}-28$ & $5.22(55) \mathrm{E}-28$ & $3.03(36) \mathrm{E}-28$ \\
\hline Ba-128 & & $6.3(30) \mathrm{E}-28$ & & \\
\hline Cs-129 & $2.28(11) \mathrm{E}-27$ & & & \\
\hline Sb-129 & $4.64(65) \mathrm{E}-27$ & $1.15(7) \mathrm{E}-27$ & $6.95(69) \mathrm{E}-28$ & \\
\hline Ba-129 & & $1.35(36) \mathrm{E}-27$ & & \\
\hline $\mathrm{I}-130$ & $2.60(29) \mathrm{E}-28$ & & $5.16(95) \mathrm{E}-28$ & $2.67(28) \mathrm{E}-28$ \\
\hline $\mathrm{I}-131$ & & $2.56(22) \mathrm{E}-27$ & $2.23(17) \mathrm{E}-27$ & $9.92(60) \mathrm{E}-28$ \\
\hline Te-132 & $1.42(17) \mathrm{E}-27$ & $2.32(11) \mathrm{E}-27$ & $1.90(8) \mathrm{E}-27$ & $9.13(90) \mathrm{E}-28$ \\
\hline $\mathrm{I}-132$ & $8.1(12) \mathrm{E}-28$ & $2.23(61) \mathrm{E}-27$ & $1.37(17) \mathrm{E}-27$ & \\
\hline Cs-132 & $1.75(12) \mathrm{E}-28$ & $2.85(45) \mathrm{E}-27$ & $2.46(14) \mathrm{E}-27$ & $1.43(23) \mathrm{E}-27$ \\
\hline La-132 & & $6.39(99) \mathrm{E}-28$ & $4.10(58) \mathrm{E}-28$ & $1.19(38) \mathrm{E}-28$ \\
\hline Ce-132 & & $5.76(44) \mathrm{E}-28$ & $2.95(56) \mathrm{E}-28$ & \\
\hline
\end{tabular}


Table 2 continued

\begin{tabular}{|c|c|c|c|c|}
\hline \multirow{2}{*}{$\begin{array}{c}\text { Residual } \\
\text { nuclei }\end{array}$} & \multicolumn{4}{|c|}{ Reaction rate $\left(\right.$ atom $^{-1} \cdot$ deuteron $\left.^{-1}\right)$} \\
\hline & 9Th & $10 \mathrm{Th}$ & $11 \mathrm{Th}$ & $12 \mathrm{Th}$ \\
\hline I-133 & $1.70(7) \mathrm{E}-27$ & $3.16(28) \mathrm{E}-27$ & $2.48(9) \mathrm{E}-27$ & $1.33(5) \mathrm{E}-27$ \\
\hline La-133 & & $1.12(23) \mathrm{E}-26$ & & \\
\hline I-134 & & $1.02(15) \mathrm{E}-26$ & $6.2(10) \mathrm{E}-27$ & $3.96(25) \mathrm{E}-27$ \\
\hline I-135 & $1.84(17) \mathrm{E}-27$ & $3.39(14) \mathrm{E}-27$ & $2.24(10) \mathrm{E}-27$ & $1.17(9) \mathrm{E}-27$ \\
\hline Xe-135 & $2.29(45) \mathrm{E}-27$ & $3.77(43) \mathrm{E}-27$ & $3.29(69) \mathrm{E}-27$ & $1.64(35) \mathrm{E}-27$ \\
\hline Cs-135m & & $1.54(21) \mathrm{E}-27$ & & \\
\hline La-135 & & $8.8(19) \mathrm{E}-27$ & & \\
\hline Ce-135 & $1.45(13) \mathrm{E}-27$ & $4.70(51) \mathrm{E}-28$ & $1.30(19) \mathrm{E}-27$ & $6.38(93) \mathrm{E}-28$ \\
\hline Cs-136 & & $4.59(24) \mathrm{E}-28$ & & \\
\hline Cs-138 & & $6.6(10) \mathrm{E}-27$ & & \\
\hline Pr-138m & & $2.77(69) \mathrm{E}-28$ & & \\
\hline Ba-139 & & & & $2.24(36) \mathrm{E}-27$ \\
\hline Ba-140 & $3.44(58) \mathrm{E}-27$ & $6.33(48) \mathrm{E}-27$ & $3.65(48) \mathrm{E}-27$ & $2.98(60) \mathrm{E}-27$ \\
\hline La-140 & $3.92(69) \mathrm{E}-28$ & $1.15(30) \mathrm{E}-27$ & $5.25(80) \mathrm{E}-28$ & $3.91(77) \mathrm{E}-28$ \\
\hline Ce-141 & & $6.07(50) \mathrm{E}-27$ & $6.30(40) \mathrm{E}-27$ & \\
\hline La-142 & $2.26(21) \mathrm{E}-27$ & & $2.92(23) \mathrm{E}-27$ & $1.19(11) \mathrm{E}-28$ \\
\hline Ce-143 & $2.10(9) \mathrm{E}-27$ & $3.50(6) \mathrm{E}-27$ & $3.07(11) \mathrm{E}-27$ & $1.57(7) \mathrm{E}-27$ \\
\hline Eu-145 & & $6.7(16) \mathrm{E}-28$ & & \\
\hline Eu-146 & & $1.02(12) \mathrm{E}-27$ & & \\
\hline Eu-147 & & $3.41(48) \mathrm{E}-27$ & & \\
\hline Gd-149 & $1.52(47) \mathrm{E}-27$ & $1.14(16) \mathrm{E}-27$ & & \\
\hline Tb-152 & & $6.61(50) \mathrm{E}-28$ & $2.76(48) \mathrm{E}-28$ & \\
\hline Dy-152 & $7.37(65) \mathrm{E}-28$ & $3.98(31) \mathrm{E}-28$ & $2.38(52) \mathrm{E}-28$ & \\
\hline Dy-155 & $8.90(44) \mathrm{E}-28$ & $6.66(22) \mathrm{E}-28$ & $3.60(37) \mathrm{E}-28$ & $1.43(24) \mathrm{E}-28$ \\
\hline Dy-157 & & $8.35(23) \mathrm{E}-28$ & $4.61(35) \mathrm{E}-28$ & $1.86(27) \mathrm{E}-28$ \\
\hline Eu-157 & $9.3(33) \mathrm{E}-28$ & & & \\
\hline Er-161 & $1.44(17) \mathrm{E}-27$ & $1.11(7) \mathrm{E}-27$ & & $3.28(62) \mathrm{E}-28$ \\
\hline Tm-167 & & $1.08(21) \mathrm{E}-27$ & & \\
\hline Tm-168 & & $1.34(24) \mathrm{E}-27$ & $2.38(47) \mathrm{E}-27$ & \\
\hline Lu-170 & & $2.52(42) \mathrm{E}-27$ & & \\
\hline Hf-170 & $1.08(8) \mathrm{E}-28$ & $1.18(21) \mathrm{E}-27$ & $5.05(67) \mathrm{E}-28$ & \\
\hline Hf-173 & & $1.09(8) \mathrm{E}-27$ & $6.60(66) \mathrm{E}-28$ & $2.68(44) \mathrm{E}-28$ \\
\hline Ta-173 & & $2.89(29) \mathrm{E}-27$ & & \\
\hline Ta-174 & & $1.89(20) \mathrm{E}-27$ & & \\
\hline Ta-175 & & & $4.43(34) \mathrm{E}-27$ & \\
\hline Yb-175 & & & & \\
\hline
\end{tabular}


Table 2 continued

\begin{tabular}{|c|c|c|c|c|}
\hline \multirow{2}{*}{$\begin{array}{l}\text { Residual } \\
\text { nuclei }\end{array}$} & \multicolumn{4}{|c|}{ Reaction rate $\left(\right.$ atom $^{-1} \cdot$ deuteron $\left.^{-1}\right)$} \\
\hline & 9Th & $10 \mathrm{Th}$ & $11 \mathrm{Th}$ & $12 \mathrm{Th}$ \\
\hline Hf-175 & & $1.39(36) \mathrm{E}-27$ & & \\
\hline Ta-176 & $9.6(27) \mathrm{E}-28$ & $1.17(12) \mathrm{E}-27$ & & \\
\hline Ta-178m & $1.21(17) \mathrm{E}-28$ & $1.21(11) \mathrm{E}-27$ & & \\
\hline Re-181 & & $1.13(9) \mathrm{E}-27$ & & \\
\hline Os-182 & & $1.14(17) \mathrm{E}-27$ & $8.61(66) \mathrm{E}-28$ & \\
\hline Re-182 & $3.23(29) \mathrm{E}-27$ & & & \\
\hline Re-183 & & $3.81(20) \mathrm{E}-27$ & & \\
\hline Os-183m & & $5.35(43) \mathrm{E}-28$ & & \\
\hline Ta-185 & & $3.27(73) \mathrm{E}-27$ & & \\
\hline Os-185 & & $1.21(10) \mathrm{E}-27$ & & \\
\hline Ir-186 & & $1.58(19) \mathrm{E}-27$ & $9.8(12) \mathrm{E}-28$ & \\
\hline Ir- $186 \mathrm{~m}$ & & & $1.50(18) \mathrm{E}-27$ & \\
\hline Pt-186 & & & $3.45(71) \mathrm{E}-28$ & \\
\hline W-187 & & $1.95(70) \mathrm{E}-27$ & & \\
\hline Ir-188 & & $1.17(27) \mathrm{E}-27$ & & \\
\hline Pt-188 & & $1.07(52) \mathrm{E}-27$ & & \\
\hline Pt-191 & & $1.53(29) \mathrm{E}-27$ & $1.30(33) \mathrm{E}-27$ & \\
\hline $\mathrm{Au}-191$ & & $2.01(27) \mathrm{E}-27$ & & \\
\hline $\mathrm{Hg}-191 \mathrm{~m}$ & & $7.3(25) \mathrm{E}-28$ & & \\
\hline $\mathrm{Au}-192$ & $1.76(22) \mathrm{E}-27$ & & $1.6(11) \mathrm{E}-27$ & \\
\hline $\mathrm{Hg}-192$ & $7.93(70) \mathrm{E}-28$ & $6.98(47) \mathrm{E}-28$ & $5.77(76) \mathrm{E}-28$ & $2.28(31) \mathrm{E}-28$ \\
\hline $\mathrm{Au}-193$ & & $3.88(54) \mathrm{E}-27$ & & \\
\hline $\mathrm{Hg}-193$ & & $1.50(42) \mathrm{E}-27$ & & \\
\hline $\mathrm{Hg}-193 \mathrm{~m}$ & & 2.73(39)E-28 & & \\
\hline Tl-194m & & $1.27(33) \mathrm{E}-27$ & & \\
\hline Ir-196m & & 5.83(89)E-28 & & \\
\hline $\mathrm{Au}-196$ & & $5.9(19) \mathrm{E}-28$ & & \\
\hline Tl-196 & $9.14(83) \mathrm{E}-28$ & $1.18(13) \mathrm{E}-27$ & $4.96(51) \mathrm{E}-28$ & $2.20(32) \mathrm{E}-28$ \\
\hline Tl-198 & $1.38(18) \mathrm{E}-27$ & $1.46(22) \mathrm{E}-27$ & & $2.98(65) \mathrm{E}-28$ \\
\hline Tl-198m & & $8.6(26) \mathrm{E}-28$ & & \\
\hline $\mathrm{Pb}-198$ & & $6.8(21) \mathrm{E}-28$ & & \\
\hline $\mathrm{Pb}-199$ & $2.87(64) \mathrm{E}-27$ & & & \\
\hline Tl-200 & $7.5(14) \mathrm{E}-28$ & & $5.07(83) \mathrm{E}-28$ & \\
\hline $\mathrm{Pb}-200$ & $5.15(65) \mathrm{E}-28$ & $6.92(46) \mathrm{E}-28$ & $3.72(34) \mathrm{E}-28$ & $1.74(41) \mathrm{E}-28$ \\
\hline $\mathrm{Pb}-201$ & & $8.22(33) \mathrm{E}-28$ & 4.74(33)E-28 & $2.11(22) \mathrm{E}-28$ \\
\hline $\mathrm{Pb}-202 \mathrm{~m}$ & & $6.2(14) \mathrm{E}-28$ & $6.22(75) \mathrm{E}-28$ & \\
\hline
\end{tabular}


Table 2 continued

\begin{tabular}{|c|c|c|c|c|}
\hline \multirow{2}{*}{$\begin{array}{c}\text { Residual } \\
\text { nuclei }\end{array}$} & \multicolumn{4}{|c|}{${\text { Reaction rate }\left(\text { atom }^{-1} \cdot \text { deuteron }^{-1}\right)}$} \\
\cline { 2 - 5 } & $9 \mathrm{Th}$ & $10 \mathrm{Th}$ & $11 \mathrm{Th}$ & $12 \mathrm{Th}$ \\
\hline Bi-202 & & $1.07(19) \mathrm{E}-27$ & & \\
\hline Po-202 & $4.50(57) \mathrm{E}-27$ & & & \\
\hline Hg-203 & & $1.30(20) \mathrm{E}-27$ & & \\
\hline Bi-203 & & $6.02(79) \mathrm{E}-28$ & & \\
\hline Po-204 & $4.21(31) \mathrm{E}-27$ & & & \\
\hline Bi-204 & $9.47(53) \mathrm{E}-28$ & $1.47(9) \mathrm{E}-27$ & $1.02(6) \mathrm{E}-27$ & $4.34(31) \mathrm{E}-28$ \\
\hline Bi-205 & & $1.30(18) \mathrm{E}-27$ & & \\
\hline Po-205 & & $1.65(42) \mathrm{E}-27$ & & \\
\hline Bi-206 & & $8.8(12) \mathrm{E}-28$ & & \\
\hline Po-207 & $9.8(15) \mathrm{E}-28$ & $1.55(14) \mathrm{E}-27$ & $8.8(15) \mathrm{E}-28$ & $2.88(60) \mathrm{E}-28$ \\
\hline At-208 & & & $5.2(12) \mathrm{E}-28$ & \\
\hline At-209 & $1.21(57) \mathrm{E}-27$ & $1.87(5) \mathrm{E}-27$ & $1.14(10) \mathrm{E}-27$ & $5.10(45) \mathrm{E}-28$ \\
\hline At-210 & & $1.24(9) \mathrm{E}-27$ & $7.74(58) \mathrm{E}-28$ & $2.89(23) \mathrm{E}-28$ \\
\hline Rn-211 & $4.82(74) \mathrm{E}-28$ & $8.74(39) \mathrm{E}-28$ & & $3.69(41) \mathrm{E}-28$ \\
\hline Bi-213 & & $3.56(23) \mathrm{E}-27$ & & \\
\hline Ac-224 & & $2.99(38) \mathrm{E}-27$ & & $8.78(69) \mathrm{E}-28$ \\
\hline Ac-226 & $1.82(21) \mathrm{E}-27$ & $3.31(7) \mathrm{E}-27$ & $2.57(29) \mathrm{E}-27$ & $1.01(6) \mathrm{E}-27$ \\
\hline Th-227 & & $4.08(49) \mathrm{E}-27$ & & \\
\hline Th-231 & $3.73(42) \mathrm{E}-26$ & $7.04(26) \mathrm{E}-26$ & $4.85(29) \mathrm{E}-26$ & $2.91(21) \mathrm{E}-26$ \\
\hline Pa-233 & $7.22(23) \mathrm{E}-26$ & $1.82(5) \mathrm{E}-25$ & $1.24(4) \mathrm{E}-25$ & $7.66(23) \mathrm{E}-26$ \\
\hline
\end{tabular}

The obtained experimental results were compared with Monte Carlo simulations performed with the FLUKA code. Ratio of the experimental and calculated reaction rates for residual nuclei ${ }^{233} \mathrm{~Pa}$ for the sample 9Th is $1.64(5)$, for $10 \mathrm{Th}$ is $1.75(5)$, for $11 \mathrm{Th}$ is $1.70(5)$, and for $12 \mathrm{Th}$ is $1.78(5)$. For residual nuclei ${ }^{231} \mathrm{Th}$, the ratio of the experimental and calculated reaction rates for the sample 9Th is $1.68(19)$, for $10 \mathrm{Th}$ is $1.95(7)$, for $11 \mathrm{Th}$ is $2.32(14)$, and for $12 \mathrm{Th}$ is $2.50(18)$. Ratio of the experimental and calculated cumulative reaction rates for such residual nuclei of fission reactions as ${ }^{87} \mathrm{Kr},{ }^{88} \mathrm{Kr},{ }^{91} \mathrm{Sr},{ }^{92} \mathrm{Sr},{ }^{92} \mathrm{Y},{ }^{93} \mathrm{Y},{ }^{97} \mathrm{Zr},{ }^{103} \mathrm{Ru}$, ${ }^{105} \mathrm{Ru},{ }^{105} \mathrm{Rh},{ }^{115} \mathrm{Cd},{ }^{128} \mathrm{Sb},{ }^{132} \mathrm{Te},{ }^{131} \mathrm{I},{ }^{132} \mathrm{I},{ }^{133} \mathrm{I},{ }^{135} \mathrm{I},{ }^{135} \mathrm{Xe},{ }^{140} \mathrm{Ba},{ }^{142} \mathrm{La}$, ${ }^{143} \mathrm{Ce}$ are in the range of $1.4-2.8$, for such residual nuclei as ${ }^{96} \mathrm{Nb},{ }^{99} \mathrm{Mo},{ }^{113} \mathrm{Ag}$, ${ }^{122} \mathrm{Sb},{ }^{124} \mathrm{Sb},{ }^{130} \mathrm{I},{ }^{135} \mathrm{Ce},{ }^{141} \mathrm{Ce}$ are in the range of 3.4-6.6. Ratio of the experimental and calculated total fission reaction rates for the sample 9Th is 2.69(39), for $10 \mathrm{Th}$ is $1.29(15)$, for $11 \mathrm{Th}$ is $1.78(19)$, and for $12 \mathrm{Th}$ is $1.87(21)$. Calculations of fission reaction rates $R(n, f)$ from the experimental data were carried out as follows. Cumulative yields $Y$ of the fission products of ${ }^{232} \mathrm{Th}$ at a neutron energy 
of $14 \mathrm{MeV}$ were taken from the TENDL-2011 [40] library. The average values of the fission product $R / Y$ ratios for the following nuclei: ${ }^{85 m} \mathrm{Kr},{ }^{87} \mathrm{Kr},{ }^{88} \mathrm{Kr}$, ${ }^{91} \mathrm{Sr},{ }^{92} \mathrm{Y},{ }^{92} \mathrm{Sr},{ }^{93} \mathrm{Y},{ }^{95} \mathrm{Zr},{ }^{96} \mathrm{Nb},{ }^{97} \mathrm{Zr},{ }^{129} \mathrm{Sb},{ }^{131} \mathrm{I},{ }^{132} \mathrm{Te},{ }^{132} \mathrm{I},{ }^{132} \mathrm{Cs},{ }^{133} \mathrm{I},{ }^{134} \mathrm{I}$, ${ }^{135} \mathrm{I},{ }^{135} \mathrm{Xe},{ }^{140} \mathrm{Ba},{ }^{141} \mathrm{Ce},{ }^{142} \mathrm{La}$, and ${ }^{143} \mathrm{Ce}$ were found to be $5.57(80) \mathrm{E}-26$ for 9Th, 9.8(11)E-26 for $10 \mathrm{Th}, 6.91(73) \mathrm{E}-26$ for $11 \mathrm{Th}$, and 3.50(39)E-26 for the sample $12 \mathrm{Th}$. These numbers are the fission reaction rates $R(n, f)$ for ${ }^{232} \mathrm{Th}$.

\section{CONCLUSIONS}

The QUINTA setup was irradiated by deuterons with energy $6 \mathrm{GeV}$. The obtained experimental results were compared with the results of simulations by the FLUKA code. By comparison of experimental and calculated data, agreements were found for residual nuclei ${ }^{233} \mathrm{~Pa},{ }^{231} \mathrm{Th}$ and for several products of fission reactions such as ${ }^{87} \mathrm{Kr},{ }^{88} \mathrm{Kr},{ }^{91} \mathrm{Sr},{ }^{92} \mathrm{Sr},{ }^{92} \mathrm{Y},{ }^{93} \mathrm{Y},{ }^{97} \mathrm{Zr},{ }^{99} \mathrm{Mo},{ }^{103} \mathrm{Ru},{ }^{105} \mathrm{Ru}$, ${ }^{105} \mathrm{Rh},{ }^{115} \mathrm{Cd},{ }^{128} \mathrm{Sb},{ }^{132} \mathrm{Te},{ }^{131} \mathrm{I},{ }^{132} \mathrm{I},{ }^{133} \mathrm{I},{ }^{135} \mathrm{I},{ }^{135} \mathrm{Xe},{ }^{140} \mathrm{Ba},{ }^{142} \mathrm{La},{ }^{143} \mathrm{Ce}$. For other products of fission reactions the ratio of the experimental and calculated cumulative reaction rates is above 3 . We suppose these ratios depend on the yield of isotopes in fission reactions induced by high-energy particles. In addition, for isomers the models are not yet able to predict the ground state/metastable level(s) split.

\section{REFERENCES}

1. P. Grand, H. Takahashi, Breeding nuclear fuels with accelerators - replacement for breeder reactors, Nuclear Instruments and Methods in Physics Research B10/11 (1985) 454-459, http://dx.doi.org/10.1016/0168-583X(85)90287-3.

2. R. A. Jameson, G. P. Lawrence, C. D. Bowman, Accelerator-driven transmutation technology for incinerating radioactive waste and for advanced application to power production, Nuclear Instruments and Methods in Physics Research B68 (1992) 474-480, http://dx.doi.org/10.1016/0168-583X(92)96126-J.

3. C. D. Bowman, E. D. Arthur, P. W. Lisowski, G. P. Lawrence, R. J. Jensen, J. L. Anderson, B. Blind, M. Cappiello, J. W. Davidson, T. R. England, L. N. Engel, R. C. Haight, H. G. Hughes III, J. R. Ireland, R. A. Krakowski, R. J. LaBauve, B. C. Letellier, R. T. Perry, G. J. Russell, K. P. Staudhammer, G. Versamis, W. B. Wilson, Nuclear energy generation and waste transmutation using an accelerator-driven intense thermal neutron source, Nuclear Instruments and Methods in Physics Research A320 (1992) 336-367, http://dx.doi.org/10.1016/0168-9002(92)90795-6.

4. F. Carminati, R. Klapisch, J.P. Revol, Ch. Roche, J. A. Rubio, C. Rubbia, An energy amplifier for cleaner and inexhaustible nuclear energy production driven by a particle beam accelerator, Tech. rep. CERN/AT/93-47, 1993,

http://cds.cern.ch/record/256520/files/at-93-047. 
5. I. Adam et al. (Energy plus Transmutation of Radioactive Wastes collaboration), The study of spatial distributions of neutron capture and fission reactions in massive target irradiated by deuterons with energies of $1-8 \mathrm{GeV}$ (QUINTA Setup): JINR preprint P1-2012-147, Dubna, 2012, 20 p., http://www1.jinr.ru/Preprints/2012/147\%28P1-2012-147\%29.pdf.

6. N. L. Asquith, S. R. Hashemi-Nezhad, S. Tyutyunnikov, M. Kadykov, V. Chilap, J. Adam, W. Furman, Activation of ${ }^{197} \mathrm{Au}$ and ${ }^{209} \mathrm{Bi}$ in a fast spectrum sub-critical assembly composed of $500 \mathrm{~kg}$ natural uranium irradiated with 1 and $4 \mathrm{GeV}$ deuterons, Annals of Nuclear Energy 63 (2014) 742-750, doi:10.1016/j.anucene.2013.09.015.

7. W.I. Furman, J.Adam, A. Baldin, et al., Recent results of the study of ADS with $500 \mathrm{~kg}$ natural uranium target assembly QUINTA irradiated by deuterons with energies from 1 to $8 \mathrm{GeV}$ at JINR Nuclotron, Proceedings of Science, XXI International Baldin Seminar on High Energy Physics Problems, September 10-15, 2012, Dubna, PoS (Baldin ISHEP XXI)086, http://pos.sissa.it/archive/conferences/173/086/Baldin\%20ISHEPP\%20XXI_086.pdf.

8. L.Zavorka, J. Adam, W. I. Furman, et al., A summary of the experimental results on reactions in the uranium samples irradiated with a deuteron beam of energies up to $8 \mathrm{GeV}$ at the QUINTA target, Proceedings of Science, XXI International Baldin Seminar on High Energy Physics Problems, September 10-15, 2012, Dubna, PoS (Baldin ISHEP XXI)089, http://pos.sissa.it/archive/conferences/173/089/Baldin\%20ISHEPP\%20XXI_089.pdf.

9. M. Suchopár, V. Wagner, I. Vrzalová, et al., Monte-Carlo simulations of natural uranium setups irradiated with relativistic deuterons by means of MCNPX code, Proceedings of Science, XXI International Baldin Seminar on High Energy Physics Problems, September 10-15, 2012, Dubna, PoS (Baldin ISHEP XXI)091, http://pos.sissa.it/archive/conferences/173/091/Baldin\%20ISHEPP\%20XXI_091.pdf.

10. L.Zavorka, J.Adam, M. Artiushenko, A. A.Baldin, V.B.Brudanin, O. Bukhal, P.Caloun, V. V.Chilap, W. I. Furman, K. Husak, M. G. Kadykov, K. Katovsky, J. Khushvaktov, I. I. Marin, V.S. Pronskikh, A. A. Solnyshkin, V. Sotnikov, V. I. Stegailov, M. Suchopar, O. Svoboda, V. M. Tsoupko-Sitnikov, S. I. Tyutyunnikov, V. Voronko, J. Vrzalova, V. Wagner, P.Zhivkov, I.Zhuk, Validation of Monte Carlo simulation of neutron production in a spallation experiment, Annals of Nuclear Energy 80 (2015) 178-187, http://dx.doi.org/10.1016/j.anucene.2015.02.010.

11. L.Zavorka, J. Adam, A. A. Baldin, P. Caloun, V. V. Chilap, W. I. Furman, M. G. Kadykov, J. Khushvaktov, V.S. Pronskikh, A. A. Solnyshkin, V. Sotnikov, V. I. Stegailov, M. Suchopar, V. M. Tsoupko-Sitnikov, S. I. Tyutyunnikov, V. Voronko, J. Vrzalova, Neutron-induced transmutation reactions in ${ }^{237} \mathrm{~Np},{ }^{238} \mathrm{Pu}$, and ${ }^{239} \mathrm{Pu}$ at the massive uranium spallation target, Nuclear Instruments and Methods in Physics Research B 349 (2015) 31-38, http://dx.doi.org/10.1016/j.nimb.2014.12.084.

12. M. Suchopár, V. Wagner, O. Svoboda, J. Vrzalová, P. Chudoba, A. Kugler, J. Adam, L.Závorka, A. Baldin, W. Furman, M.Kadykov, J. Khushvaktov, A. Solnyshkin, V. Tsoupko-Sitnikov, S. Tyutyunnikov, Cross-section studies of relativistic deuteron reactions on copper by activation method, Nuclear Instruments and Methods in Physics Research B 344 (2015) 63-69, http://dx.doi.org/10.1016/j.nimb.2014.12.006. 
13. J. Adam, Chitra Bhatia, K. Katovsky, V. Kumar, M. Majerle, V.S.Pronskikh, A.M. Khilmanovich, B.A. Martsynkevich, I. V.Zhuk, V.M. Golovatiouk, W. Westmeier, A. A. Solnyshkin, V.M. Tsoupko-Sitnikov, A. S. Potapenko, A study of rates of $(n, f),(n, \gamma)$, and $(n, 2 n)$ reactions in ${ }^{\text {nat }} \mathrm{U}$ and ${ }^{232} \mathrm{Th}$ produced by the neutron fluence in the graphite setup (GAMMA-3) irradiated by $2.33 \mathrm{GeV}$, Eur. Phys. J. A (2011) 47:85, http://dx.doi.org/10.1140/epja/i2011-11085-4.

14. N. L. Asquith, S. R. Hashemi-Nezhad, W. Westmeier, I. Zhuk, S. Tyutyunnikov, J. Adam, Study of ${ }^{232} \mathrm{Th}(n, \gamma)$ and ${ }^{232} \mathrm{Th}(n, f)$ reaction rates in a graphite moderated spallation neutron field produced by $1.6 \mathrm{GeV}$ deuterons on lead target, Nuclear Instruments and Methods in Physics Research B 344 (2015) 51-58, http://dx.doi.org/10.1016/j.nimb.2014.12.004.

15. M. Majerle, J. Adam, P. Čaloun, S. A. Gustov, V. Henzl, D. Henzlová, V. G. Kalinnikov, M. I. Krivopustov, A. Krása, F. Kř́̌žek, A. Kugler, I. V. Mirokhin, A. A. Solnyshkin, V. M. Tsoupko-Sitnikov, V. Wagner, Spallation experiment on thick lead target: Analysis of experimental data with Monte Carlo codes. JINR Preprint E15-2008-94, Dubna, 2008. http://www1.jinr.ru/Preprints/2008/094\%28E15-2008-94\%29.pdf.

16. J. Adam, A. Balabekyan, R. Brandt, et al., Investigation of the formation of residual nuclei in reactions induced by $660 \mathrm{MeV}$ protons interacting with the radioactive ${ }^{237} \mathrm{~Np}$, ${ }^{241} \mathrm{Am}$ and ${ }^{129} \mathrm{I}$ targets, Journal of Nuclear Science and Technology, Supplement 2, p. 272-275 (August 2002), http://dx.doi.org/10.1080/00223131.2002.10875092.

17. V.Henzl, D. Henzlova, A. Kugler, V. Wagner, J. Adam, P.Caloun, V. G. Kalinnikov, M. I. Krivopustov, A. V.Pavliouk, V. I. Stegajlov, V. M. Tsoupko-Sitnikov, W. Westmeier, Transmutation of ${ }^{129} \mathrm{I}$ with high energy neutrons produced in spallation reactions induced by protons in massive target, Journal of Nuclear Science and Technology, Supplement 2, p. 1248-1251 (August 2002), http://dx.doi.org/10.1080/00223131.2002.10875330.

18. T. T. Böhlen, F. Cerutti, M.P.W.Chin, A. Fassó, A. Ferrari, P. G. Ortega, A. Mairani, P. R. Sala, G. Smirnov and V. Vlachoudis, The FLUKA code: Developments and challenges for high energy and medical applications, Nuclear Data Sheets 120 (2014) 211-214, http://dx.doi.org/10.1016/j.nds.2014.07.049.

19. A. Ferrari, P.R.Sala, A. Fassó, J.Ranft, FLUKA: A multi-particle transport code, CERN-2005-10 (2005), INFN/TC_05/11, SLAC-R-773, http://slac.stanford.edu/pubs/slacreports/reports16/slac-r-773.pdf.

20. J.Frana, Program DEIMOS32 for gamma-ray spectra evaluation, Journal of Radioanalytical and Nuclear Chemistry, Vol.257, No.3 (2003) 583-587, http://link.springer.com/article/10.1023\%2FA\%3A1025448800782.

21. I. Adam, V.S. Pronskikh, A. R. Balabekyan, V.G. Kalinnikov, J. Mrazek, A. N. Priemyshev, J. Frána, Program package and supplements to activation analysis for calculations of nuclear reaction cross-sections, Measurement Techniques, No. 1 (2001) 57-61, JINR Preprint P10-2000-28, Dubna, 2000, http://www1.jinr.ru/Preprints/2000/p10-2000-28.pdf

22. S. Sudár, "TRUECOINC", a software utility for calculation of the true coincidence correction, IAEA-TECDOC-1275, p. 37-48, http://www.iaea.org/inis/collection/ NCLCollectionStore/_Public/33/017/33017174.pdf?r=1. 
23. I. Adam, A. Balbekyan, V. S. Barashenkov et al. Study of product formation in protonnuclear reactions on the ${ }^{129} \mathrm{I}$ target induced by $660-\mathrm{MeV}$ protons, Part. Nucl., Lett., 2004. V. 1, No.4(121). P. 53-64, http://www1.jinr.ru/Pepan_letters/panl_4_2004/06_adam.pdf.

24. J. Adam, K. Katovsky, M. Majerle, M. I. Krivopustov, V. Kumar, Chitra Bhatia, Manish Sharma, A. A. Solnyshkin, V. M. Tsoupko-Sitnikov, A study of nuclear transmutation of $\mathrm{Th}$ and ${ }^{\text {nat }} \mathrm{U}$ with neutrons produced in $\mathrm{Pb}$ target and $\mathrm{U}$ blanket irradiated by $1.6 \mathrm{GeV}$ deuterons, Eur. Phys. J. A 43 (2010) 159-173, JINR Preprint E15-2008-118, Dubna, 2008, http://link.springer.com/article/10.1140/epja/i2010-10909-y.

25. A. Ferrari, P. R. Sala, A new model for hadronic interactions at intermediate energies for the FLUKA code // Proc. MC93 Int. Conf. on Monte Carlo Simulation in High Energy and Nuclear Physics, Tallahassee (Florida), 22-26 February 1993. Ed. by Dragovitsch P., Linn S.L., Burbank M., World Scientific, Singapore 1994, p. 277-288, http://www.fluka.org/content/publications/1993_mc_preq.pdf.

26. A. Ferrari, P.R. Sala, The Physics of High Energy Reactions, Proc. Workshop on Nuclear Reaction Data and Nuclear Reactors Physics, Design and Safety, International Centre for Theoretical Physics, Miramare-Trieste, Italy, 15 April - 17 May 1996, http://www.fluka.org/content/publications/1996_trieste.pdf.

27. A. Ferrari, J.Ranft, S. Roesler, P.R. Sala, The production of residual nuclei in peripheral high energy nucleus-nucleus interactions, Z. Phys. C 71 (1996) 75-86, http://link.springer.com/article/10.1007\%2Fs002880050149.

28. A. Ferrari, J. Ranft, S. Roesler, P. R. Sala, Cascade particles, nuclear evaporation, and residual nuclei in high energy hadron-nucleus interactions, Z. Phys. C 70 (1996) 413-426, http://link.springer.com/article/10.1007\%2Fs002880050119.

29. Barashenkov V.S., Toneev V.D. Interactions of High Energy Particles and Nuclei with Nuclei, Moscow, Atomizdat (1972) (in Russian).

30. H. J. Möhring, Hadron-nucleus inelastic cross-sections for use in hadron cascade calculations at high energies, CERN TIS-RP/116 (1983), https://www.researchgate.net/ publication/29514654_Hadron-nucleus_inelastic_cross-sections_for_use_in_hadroncascade_calculations_at_high_energies.

31. Qing-biao Shen, Systematics of intermediate energy proton nonelastic and neutron total cross section, IAEA Report INDC(CPR)-020, 1991, https://inis.iaea.org/search/search.aspx?orig_q=RN:22082926.

32. R. E. Prael, A. Ferrari, R. K. Tripathi, A. Polanski, Comparison of nucleon cross section parameterization methods for medium and high energies, Proc. 4th Workshop on Simulating Accelerator Radiation Environments (SARE4), 14-16 September 1998, Knoxville (Tenn.).

33. R. E. Prael, A. Ferrari, R. K. Tripathi, A. Polanski, Plots supplemental to: "Comparison of nucleon cross section parameterization methods for medium and high energies", Los Alamos report LA-UR-98-5843 (1998).

34. H. Sorge, H. Stcöker, W. Greiner, Poincaré invariant Hamiltonian dynamics: Modelling multi-hadronic interactions in a phase space approach, Annals of Physics 192 (1989) 266-306,

http://www.sciencedirect.com/science/article/pii/000349168990136X. 
35. H. Sorge, H. Stöcker, W. Greiner, Relativistic quantum molecular dynamics approach to nuclear collisions at ultrarelativistic energies, Nuclear Physics A498 (1989) 567c-576c, http://www.sciencedirect.com/science/article/pii/0375947489906416.

36. H. Sorge, Flavor production in $\mathrm{Pb}(160 \mathrm{~A} \mathrm{GeV})$ on $\mathrm{Pb}$ collisions: Effect of color ropes and hadronic rescattering, Physical Review C 52 (1995) 3291-3314, http://journals.aps.org/prc/abstract/10.1103/PhysRevC.52.3291.

37. M.Cavinato, E. Fabrici, E. Gadioli, E. Gadioli Erba, E. Galbiati, Monte Carlo calculations using the Boltzmann Master Equation theory of nuclear reactions, Physics Letters B 382 (1996) 1-5, http://www.sciencedirect.com/science/article/pii/0370269396006521.

38. M. Cavinato, E. Fabrici, E. Gadioli, E. Gadioli Erba, G. Riva, Monte Carlo calculations of heavy ion cross-sections based on the Boltzmann Master equation theory, Nuclear Physics A 679 (2000) 753-764, http://www.sciencedirect.com/science/article/pii/S0375947400003572.

39. F. Cerutti, G. Battistoni, G. Capezzali, P.Colleoni, A. Ferrari, E. Gadioli, A. Mairani, A. Pepe, Low energy nucleus-nucleus reactions: The BME approach and its interface with FLUKA, Proc. 11th International Conference on Nuclear Reaction Mechanisms, Varenna (Italy), June 12-16, 2006,

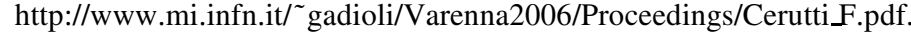

40. TENDL-2011, Nuclear data library www.talys.eu/.

41. K. Nakamura et al. (Particle Data Group), J. Phys. G 37 (2010) 075021, http://pdg.lbl.gov/2010/pdg_2010.html.

Received on September 23, 2016. 


\section{Редактор Е. И. Кравченко}

Подписано в печать 27.10.2016.

Формат $60 \times 90 / 16$. Бумага офсетная. Печать офсетная.

Усл. печ. л. 1,44. Уч.-изд. л. 2,03. Тираж 215 экз. Заказ № 58935.

Издательский отдел Объединенного института ядерных исследований 141980 , г. Дубна, Московская обл., ул. Жолио-Кюри, 6.

E-mail: publish@jinr.ru www.jinr.ru/publish/ 